Original paper

\title{
On the interplay effect for moving targets treated with the CyberKnife static tracking system
}

\author{
Michele Zeverino ${ }^{a}$, Yihan Jia ${ }^{\mathrm{b}}$, Leo Charosky ${ }^{\mathrm{a}}$, Jean Bourhis ${ }^{\mathrm{c}}$, Francois O. Bochud ${ }^{\mathrm{a}}$, \\ Raphael Moeckli ${ }^{\text {a, }}$ \\ ${ }^{a}$ Institute of Radiation Physics, Lausanne University Hospital and Lausanne University, Lausanne, Switzerland \\ ${ }^{\mathrm{b}}$ École polytechnique fédérale de Lausanne- EPFL, Lausanne, Switzerland \\ ${ }^{\mathrm{c}}$ Radiation Oncology Department, Lausanne University Hospital and Lausanne University, Lausanne, Switzerland
}

\section{A R T I C L E I N F O}

\section{Keywords:}

Interplay effect

CyberKnife static tracking technique

Lung SBRT

Gafchromic EBT3

\begin{abstract}
A B S T R A C T
Purpose: To assess the interplay effect amplitude between different planned MU distributions and respiratory patterns in the CyberKnife system when treating moving targets with static tracking technique.

Methods: Small- and Large-Respiratory Motions (SRM and LRM) differing in amplitude and frequency were simulated in a semi-anthropomorphic dynamic thorax phantom. The interplay effect was evaluated for both respiration motions in terms of GTV coverage and conformity for three plans designed with an increasing range of MU per beam (small, medium and large). Each plan was delivered three times changing the initial beam-on phase to assess the inter-fraction variation. Dose distributions were measured using radiochromic films placed in the GTV axial and sagittal planes.

Results: Generally, SRM plans gave higher GTV coverage and were less dependent on beam-on phases than LRM plans. For SRM (LRM) plans, the GTV coverage ranged from $95.2 \%$ to $99.7 \%$ (85.9\% to 99.8\%). Maximum GTV coverage was found for large MU plans in SRM and for small MU plans in LRM. Minimum GTV coverage was found for medium MU plans for both SRM and LRM. For SRM plans, dose conformity decreased with increasing MU range while the variation was reduced for LRM plans. Large MU plans reduced the inter-fraction variation for SRM and LRM.

Conclusions: We confirmed the interplay effect between target motion and beam irradiation time for CyberKnife static tracking. Plans with large MU per beam improved the GTV coverage for small motion amplitude and the inter-fraction dose variation for large motion amplitude.
\end{abstract}

\section{Introduction}

Small lung tumors with respiratory correlated motion can be treated with the CyberKnife ${ }^{\mathrm{TM}}$ (CK; Accuray Incorporated, Sunnyvale, CA, USA) using real-time tumor tracking techniques such as fiducial markersbased tracking with Synchrony ${ }^{\mathrm{TM}}$ or fiducial-free tracking with XSight Lung Tracking ${ }^{\mathrm{TM}}$ (XLT) [1-7]. Both tracking techniques use a correlation model between respiratory phase and tumor position. That model is generated by the continuous acquisition of respiratory signals matched with continually updated $\mathrm{kV}$ stereoscopic image pairs of fiducial markers or tumor tissue density for Synchrony or XLT, respectively. The correlation model is then used during treatment to predict the tumor position and drive the robotic arm to track the rigid target movement accordingly.
Nonetheless, both tracking techniques have several limitations. For Synchrony, the implantation of fiducial markers presents risks of pneumothorax [8], fiducial markers can also be misplaced or they can migrate from their original position, resulting in sub-optimal treatments or delays [9]. XLT requires that tumors have a minimum diameter of 15 $\mathrm{mm}$ [10] although larger sizes provided best results (tumors larger than $35 \mathrm{~mm}$ were successfully tracked more than $80 \%$ of the time[11]). Tumors also need to be located at least $15 \mathrm{~mm}$ away from any bone structure with their projections not aligned at 45 degrees to the spine [12]. Finally, XLT requires adequate tumor density in order for the system to visualize it [10].

When neither of these two dynamic tracking techniques can be used because of the aforementioned issues, an indirect tracking technique combined with a motion management strategy is a possible alternative

\footnotetext{
* Corresponding author.

E-mail address: raphael.moeckli@chuv.ch (R. Moeckli).
} 


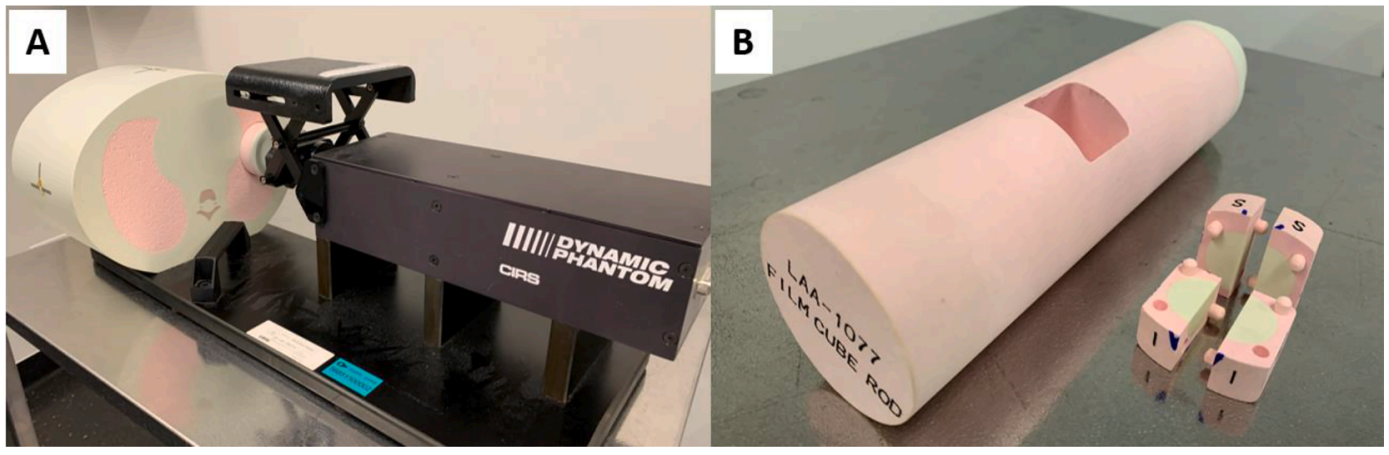

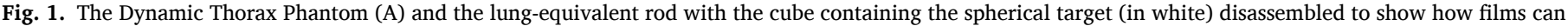
be inserted (B).

[7]. The XSight Spine Tracking ${ }^{\mathrm{TM}}$ (XST) static tracking technique is intended to track displacements of skeletal structures such as the vertebrae, while correcting for patient displacements in 6 degrees of freedom (translations and rotations). In principle, the vertebra adjacent to the moving tumor is used as a surrogate for the whole patient alignment. An 81-node grid region of interest (ROI) is typically centered on the adjacent vertebra when the plan is created to then be used by the tracking algorithm during delivery in order to compute and correct for displacements of nodes between reference and daily images. X-ray images are taken at time intervals ranging from $15 \mathrm{~s}$ to $60 \mathrm{~s}$.

Since the target motion is not taken into account by the tracking algorithm, using the XST technique for moving tumors necessitates including the whole target motion within the irradiation volume by defining an Internal Target Volume (ITV) based on the Gross Tumor Volume (GTV) contoured on the 4D image dataset and a Planning Target Volume (PTV) that accounts for systematic and random errors on the reference 3D respiration CT phase [13]. The specificity of the CK in this context is the use of a large number of small beams with each one only partially irradiating the PTV allowing for dose gradients steeper than any other ITV- and linac-based technique and consequent dose reduction to normal tissues. As the tumor is not tracked in real time, the GTV will receive full, partial, or even no primary dose from a given beam because of the interplay effect between tumor motion and collimator movement. This means that even an excellent planned coverage of the PTV may not guarantee a sufficient GTV coverage. The amplitude of this kind of geographical miss depends not only on the irradiation technique and its intrinsic planning parameters, but also on the irradiation beam start time with respect to the respiratory phase [14]

The typical planning parameters that can affect the interplay effect in this specific context are the beam collimator size(s), the number of collimators, the isodose prescription, and the dose per fraction. Previous studies have already investigated the interplay effect between planning parameters and tumor motion patterns differing in amplitude and frequency and have shown limitations when using the XST technique with moving tumors $[15,16]$.

However, to our knowledge, no studies have yet investigated the direct role of the number of Monitor Units (MU) per beam $\left(\mathrm{MU}_{\mathrm{pb}}\right)$ on the interplay effect. The $\mathrm{MU}_{\mathrm{pb}}$ range can be varied in the Precision TPS (Accuray Inc., Sunnyvale, CA, USA) setting the minimum and maximum number of $\mathrm{MU}_{\mathrm{pb}}$. These parameters define the minimum and maximum number of MU delivered by a single beam in a certain position with a defined collimator size. Hence, the choice of this parameter determines the planned MU distribution for each collimator size used. As MU is by definition a surrogate of irradiation time, different planned MU distributions will have a different impact on the delivered dose distribution, because of the different interplay effect between beam irradiation time and tumor motion.

The aim of this study was to provide the evidence and the evaluation of the magnitude of this specific interplay effect for the CK when treating
Table 1

Details of respiratory motion patterns. $A_{A P}, A_{L R}$ and $A_{S I}$ denote the peak-to-peak displacement range of tumor center on nominal AP, LR and IS axis, respectively.

\begin{tabular}{lllll}
\hline \multirow{2}{*}{ Motion Type } & \multicolumn{2}{l}{ Motion parameters } & \\
& $A_{A P}(\mathrm{~mm})$ & $A_{L R}(\mathrm{~mm})$ & $A_{S I}(\mathrm{~mm})$ & $T(\mathrm{~s})$ \\
\hline SRM & 2 & 3 & 10 & 3 \\
LRM & 4 & 6 & 40 & 6 \\
\hline
\end{tabular}

moving targets by means of an indirect and static tracking technique. We tested the impact of three different planned MU distributions on the intra- and inter- fraction delivered dose to the tumor volume for two typical breathing cycles.

\section{Materials and methods}

\subsection{Moving phantom setup}

The Dynamic Thorax Phantom (DTP; CIRS Inc.; Norfolk, VA, USA) used in this study was designed with three different types of epoxy resins that are radio-equivalent to soft tissue, lung, and spine. It featured a moving lung-like rod equipped with a spherical target $(\varnothing=25 \mathrm{~mm})$ for which films can be placed orthogonally in the axial and sagittal planes [17].

The DTP was mounted on a two-axis motor enabling threedimensional movement (see Fig. 1). The center of the spherical target was off-centered from the axis of the rod so that rotations induced left-right (LR) and anterior-posterior (AP) movements whereas the longitudinal axis of the motor provided movement in the inferiorsuperior (IS) direction. Therefore, sagittal film orientations continuously change during irradiation due to continuous trans-rotational motion of the target. The linear and angular motion accuracies were 0.05 $\mathrm{mm}$ and 0.2 degrees, respectively [18].

Small respiratory motion (SRM) and large respiratory motion (LRM) were designed based on $\cos ^{4}$ function that provides the highest correlation with patient respiratory motion [19]. Both SRM and LRM were modeled by the function:

$\boldsymbol{x}(t)=\boldsymbol{x}_{0}+\boldsymbol{A} \cos ^{4}\left(t \cdot \frac{\pi}{\mathrm{T}}\right)$

Where $x_{0}$ is the initial position (end inhale), $\boldsymbol{A}$ is the amplitude vector given by $A_{L R} ; A_{A P} ; A_{S I}[\mathrm{~mm}]$, and $\mathrm{T}$ is the period [s].

SRM had the shortest breath interval and smallest thoracic expansion mimicking a fast and shallow respiration, while LRM had the slowest and deepest respiration producing the largest tumor motion amplitude. Table 1 summarizes the parameters used for SRM and LRM. 


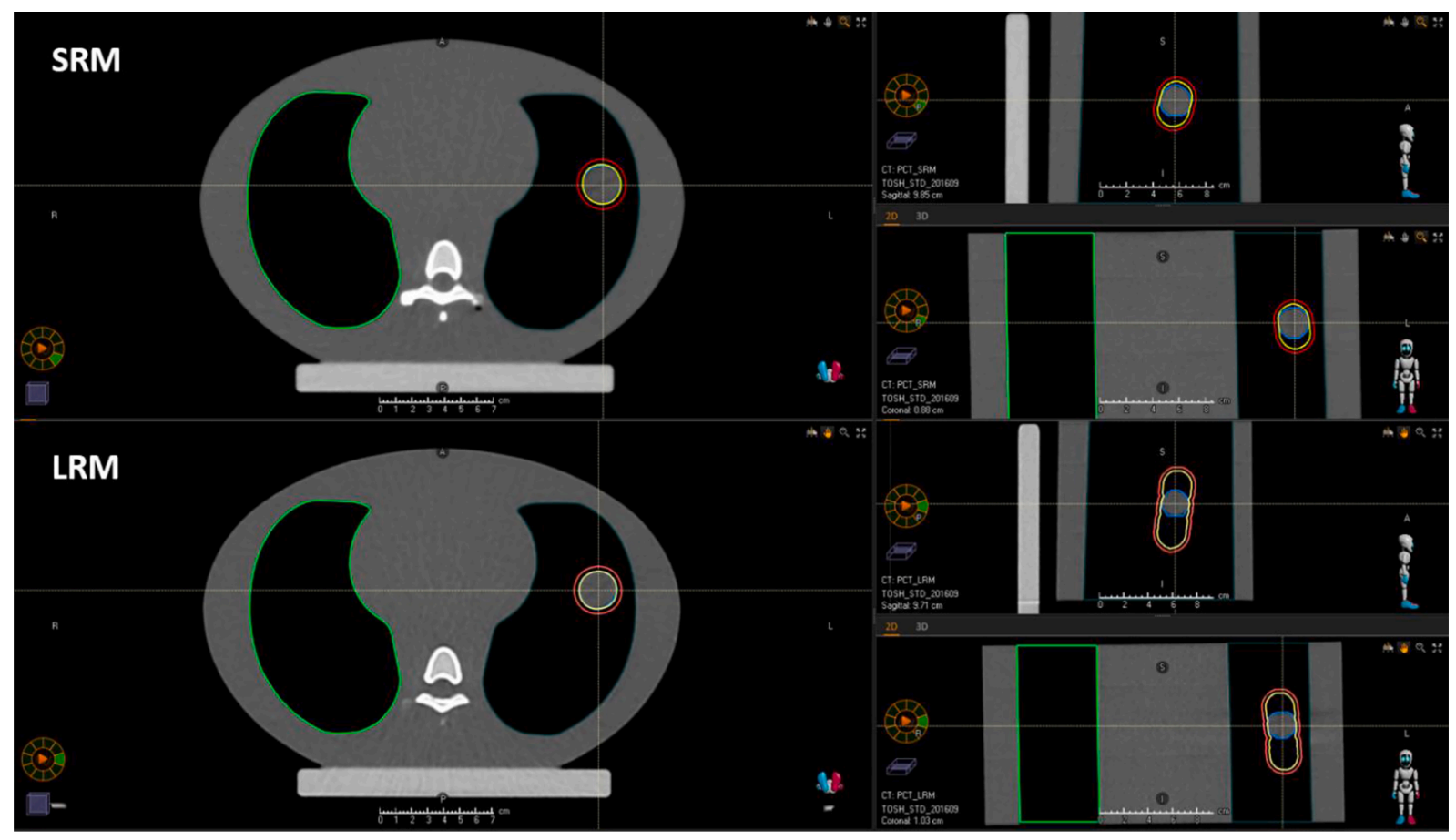

Fig. 2. The DTP CT contoured for SRM (upper panel) and LRM (lower panel). The volumes shown are: Blue $=$ GTV, Yellow $=$ ITV, Red $=$ PTV. (For interpretation of the references to color in this figure legend, the reader is referred to the web version of this article.)

\section{2. $4 D C T$ simulation, target definition and ITV determination}

Four-dimensional CT images of $1.0 \mathrm{~mm}$ thickness were acquired on an Aquilion LB CT scanner (Canon Medical Systems Europe) together with the AZ-733 V respiratory gating system (Anzai Medical, Tokyo, Japan) with the belt respiratory sensor mounted on the chest plate of the DTP. For each 4DCT dataset, 10 equally time-binned 3D-CT datasets were extracted and transferred to Raystation v9.B TPS (RaySearch Laboratories, Stockholm, Sweden).

The spherical target was contoured on each phase and defined as GTV. ITV was defined on the mid-ventilation CT phase as the union of the GTVs obtained in each CT phase providing the position of the GTV closest to its mid-position [18]. A margin of $3 \mathrm{~mm}$ was added in each direction to the ITV to generate the PTV in line with our current clinical practice (see Fig. 2). The planning was performed in the Precision TPS.

\subsection{Treatment planning optimization and plan delivery}

The XST technique integrated with the CK system necessitated defining the ROI-node grid to be tracked. We used an $80 \mathrm{~mm}$ high ROI, centered so that the full spine region adjacent to the PTV was symmetrically encompassed in the $x$ and $y$ axis and was at the same height as the geometric center of PTV along the $\mathrm{z}$ axis. IRIS-based collimators of 25 $\mathrm{mm}$ and $35 \mathrm{~mm}$ were used for both SRM and LRM cases. These two collimator dimensions represented a trade-off between a good dose conformity (25 mm diameter as the GTV dimension) and a minimal treatment time (35 $\mathrm{mm}$ diameter)

The optimization process was carried out with the VOLO ${ }^{\mathrm{TM}}$ algorithm [20]. The prescription dose of $60 \mathrm{~Gy}(7.5 \mathrm{~Gy} /$ fraction) was initially optimized using the Ray-Tracing (RT) algorithm (type A) in order to cover at least $98 \%$ of the PTV with the prescribed dose, and then Monte Carlo (MC) algorithm (type B) was used to recalculate and rescale the dose to ensure $100 \%$ coverage of the GTV with the prescribed dose. The rescaling consisted of lowering the percentage isodose prescription once the dose was recalculated with MC. We followed the method proposed by Lacornerie et al [21] avoiding the prescription to the PTV when type B dose calculation algorithms (i.e. Monte Carlo) are used for fluence optimization.

Three different configurations in terms of minimum and maximum number of $\mathrm{MU}_{\mathrm{pb}}$ were tested:

- Small MU (sMU) plan: $6 \leq \mathrm{MU}_{\mathrm{pb}}$ per fraction $\leq 62.5$ (i.e. $48 \leq \mathrm{MU}_{\mathrm{pb}}$ per plan $\leq 500)$;

- Medium MU (mMU) plan: $30 \leq \mathrm{MU}_{\mathrm{pb}}$ per fraction $\leq 90$ (i.e. $180 \leq$ $\mathrm{MU}_{\mathrm{pb}}$ per plan $\leq 720$ );

- Large MU (lMU) plan: $60 \leq \mathrm{MU}_{\mathrm{pb}}$ per fraction $\leq 120$ (i.e. $360 \leq \mathrm{MU}_{\mathrm{pb}}$ per plan $\leq 960$ ).

The sMU plan configuration was also what would have been used for a similar real case in the clinical setting. All remaining planning parameters were kept fixed for both SRM and LRM cases: the maximum number of nodes of 117 (corresponding to its maximum value) and a MU penalty factor [20] of 1 (no attempt made from optimizer to decrease or increase the planned number of MU). Each plan was delivered to the DTP three times with beam-on synchronized with the initial respiratory phase being end inhale (I), end exhale (E) and mid-ventilation (M). Each time the DTP was repositioned to the table to evaluate also the interfraction dose variation. We performed a total of 18 irradiations.

\subsection{Film dosimetry and analysis}

We evaluated the delivered dose using Gafchromic EBT3 films (Ashland Advanced materials, Bridgewater, NJ, USA; type XLT, lot number $02122002 \mathrm{X}$ ). The size of the films was $3.175 \times 3.175 \mathrm{~cm}^{2}$ and they were precut in pairs to fit the spherical target corresponding to the initial axial (AL) and sagittal (AS) orientations, respectively. Since the films were delivered precut without any information about the orientation of the sensitive layer and because scan response of EBT3 depends on the film orientation on the scanner [22], they were all scanned before irradiation to check for consistency (with no impact on the darkening). Films were scanned (Epson V700, Seiko Epson Corporation, Japan) $15 \mathrm{~h}$ and $39 \mathrm{~h}$ after irradiation to obtain two different post-irradiation calibrations. The film scanning was performed with a resolution of 150 dots per inch, all image enhancement filters turned off and in positive 48 bits 
Table 2

Comparison of number of beams and mean $\mathrm{MU}_{\mathrm{pb}}$ per fraction. Standard deviation for the mean $\mathrm{MU}_{\mathrm{pb}}$ value is reported in brackets.

\begin{tabular}{|c|c|c|c|c|c|c|}
\hline & \multicolumn{3}{|c|}{$\begin{array}{l}\text { Number of beams per } \\
\text { fraction }\end{array}$} & \multicolumn{3}{|c|}{ Mean (standard deviation) $\mathrm{MU}_{\mathrm{pb}}$ per fraction } \\
\hline & sMU & $\mathrm{mMU}$ & IMU & sMU & $\mathrm{mMU}$ & IMU \\
\hline SRM & 73 & 28 & 14 & $26.9(19.0)$ & 74.0 (19.9) & 137.9 (15.3) \\
\hline LRM & 143 & 51 & 25 & 21.6 (13.9) & $59.9(19.8)$ & $112.9(16.5)$ \\
\hline
\end{tabular}

colors. Films were scanned in the inner region of the flat bed by means of a positioning frame in order to minimize the inter-scan variability.

Films were calibrated in absorbed dose to water using a $6 \mathrm{MV}$ photon beam of an Elekta Synergy linear accelerator (Elekta AB, Stockholm, Sweden) at $5 \mathrm{~cm}$ water equivalent depths with dose levels ranging from 0.5 to $15 \mathrm{~Gy}$ for a total of 10 films including the zero dose level. The protocol we currently use for film absorbed dose calibration lead to an uncertainty of $4 \%$ [23].

The TIFF images of scanned films were processed in MATLAB. The red channel of the images was extracted from the RGB image to increase the calibration curve sensitivity [22]. A circular ROI $(\varnothing=25 \mathrm{~mm})$ placed on the film center was superposed to every image to mimic the central projections of the GTV. The intersection of the film region and the circular tumor was defined as GTV on both AL and AS films.

By analogy with DVH in three dimensions, relative and absolute dose-area histogram (DAH) in two dimensions were computed for the whole film and the GTV. The GTV coverage was defined as the volume (\%) of the GTV receiving 7.5 Gy. The dose conformity was calculated using the new conformity index (nCI) defined as:

$n C I=\frac{A_{G T V} \cdot A_{f u l l}, 7.5 G y}{A_{G T V, 7.5}^{2} G y}$

Where $A_{\text {GTV }}$ is the area of GTV, $A_{\text {full, } 7.5 \mathrm{~Gy}}$ the area of full film receiving 7.50 Gy, and $A_{\mathrm{GTV}, 7.5 \mathrm{~Gy}}$ the area of GTV receiving $7.50 \mathrm{~Gy}$. A nCI of 1 means a complete conformity between the isodose of the prescribed dose and the GTV. For a spherical target, a good conformity should result in $\mathrm{nCI}$ less than 1.1, while for more complex or elongated targets, values of up to 1.4 can be considered acceptable [24]. The GTV coverage and the nCI were obtained from the computed DAHs.

\section{Results}

\subsection{Treatment planning optimization}

Table 2 summarizes the number of beams and the mean value of $\mathrm{MU}_{\mathrm{pb}}$ per fraction for the three plans tested and two respiratory motions.

As expected, the average of MU systematically increased with the range of MU set in the plan for both respiratory motions. Plans with larger $\mathrm{MU}_{\mathrm{pb}}$ led to fewer beam numbers. The mean $\mathrm{MU}_{\mathrm{pb}}$ value was found to be systematically lower for LRM due to the increased number of beams.

Cumulative histograms (over the 8 fractions) of $\mathrm{MU}_{\mathrm{pb}}$ for $\mathrm{sMU}$, $\mathrm{mMU}$, and IMU plans are presented in Fig. 3 for SRM and LRM. Both minimum and maximum $\mathrm{MU}_{\mathrm{pb}}$ values were higher than the set values. This was due to the rescaling of the dose once MC was used for recalculation. The MU distribution comparison between RT and MC algorithms are reported in Fig. 1s of the Supplementary Material.

For sMU plans, the optimization algorithm maximized the beams with less MU providing a non-uniform MU distribution. For mMU and IMU plans, $\mathrm{MU}_{\mathrm{pb}}$ distributions were found to be more uniform, in particular for LRM. The analysis of the MU histograms sorted by collimator size (see Figs. $2 \mathrm{~s}$ and $3 \mathrm{~s}$ of the Supplementary Material) suggested a different use of collimators between the respiratory patterns: the averaged MU value per collimator was found to be similar for SRM while it was higher for $35 \mathrm{~mm}$ than $25 \mathrm{~mm}$ for LRM.

\subsection{Film analysis}

The sensitive layer orientation was found to be consistent among the films (less than $2 \%$ ) as declared by the manufacturer once the anterior label (A) was aligned with the long axis of the scanner for both AL and AS films.

Dose distributions measured in the central sections of the GTV for the SRM case are reported in Figs. 4 and 5 for AL and AS films, respectively.

AL films placed on the axial central section of the GTV never reached the edge of the ITV. For these films dose distributions were quite symmetric with respect to the GTV central axis for all different MU plans. AS films instead provided dose distribution rotating along the sagittal axis, the SI edges of the films were expected to reach the edges of the ITV in both directions. In this case, the dose distribution (see the $750 \mathrm{cGy}$ for instance in Fig. 5) resulted asymmetric in the SI direction showing a shift towards the inferior side that tend to reduce with the increasing MU/ beam. In both planes, dose increased according to the number of $\mathrm{MU}_{\mathrm{pb}}$. The mid phase resulted in a lower dose delivered to the GTV in both planes.

Similarly, for the LRM case, dose distributions are reported in Figs. 6 and 7 for AL and AS films, respectively. Here the interplay effect was more pronounced and difficult to describe as measured dose distributions varied differently between MU plans through the different respiratory phase in both planes. Although reduced in its amplitude, the asymmetry of the dose distribution in the AS films was still observed similarly to the SRM case.

DAH of SRM and LRM are reported in Figs. $4 \mathrm{~s}$ and 5s, respectively, in the Supplementary Material. The results for the GTV coverage of different MU plans for both SRM and LRM cases are summarized in
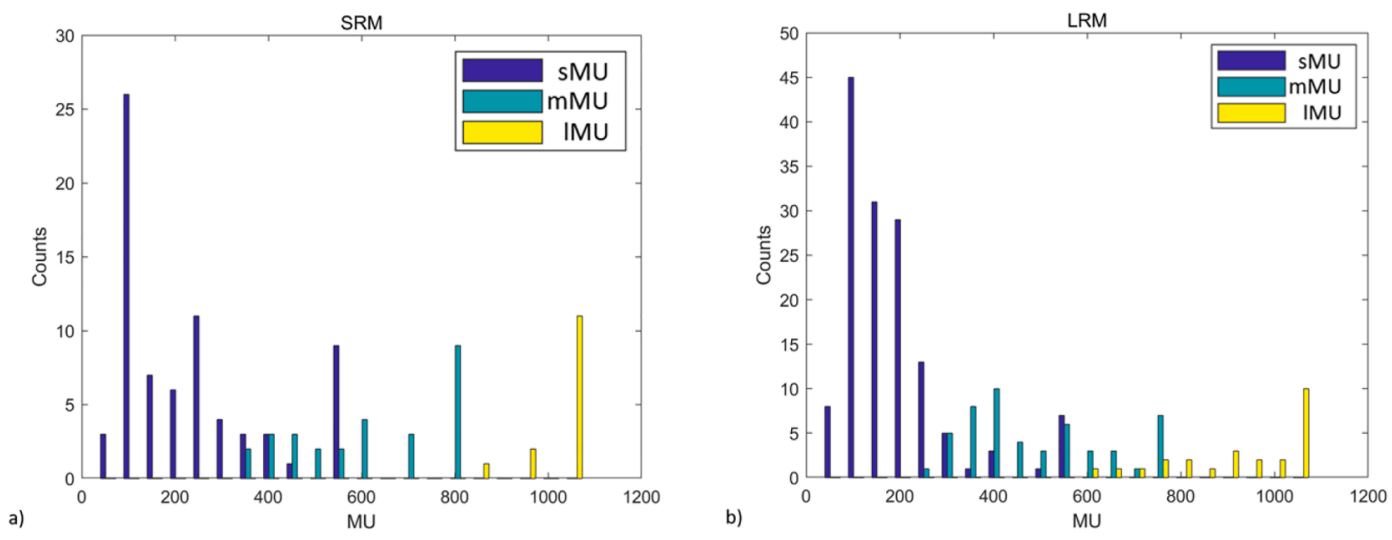

Fig. 3. $\mathrm{MU}_{\mathrm{pb}}$ distribution obtained from different MU plans for SRM (a) and LRM (b). Values reported are relative to the total number of fractions (i.e. the plan) and relative to the MC dose calculation. 


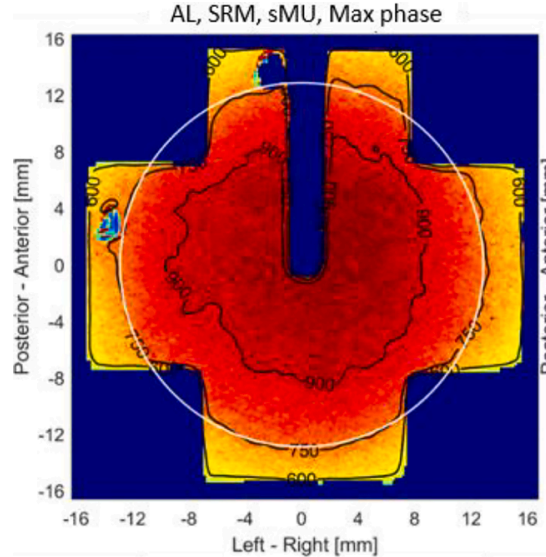

AL, SRM, sMU, Mid phase

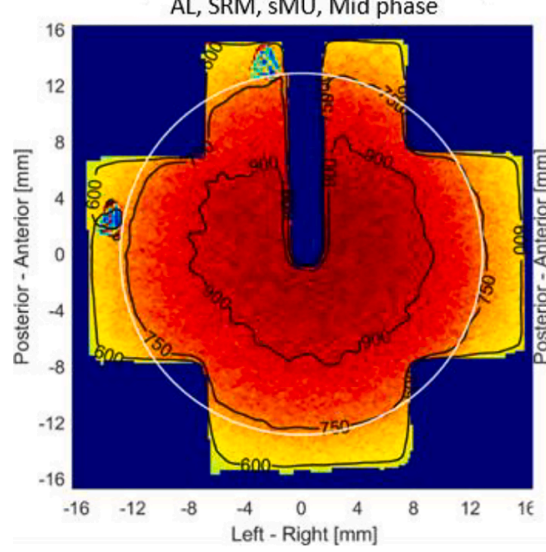

AL, SRM, sMU, Min phase

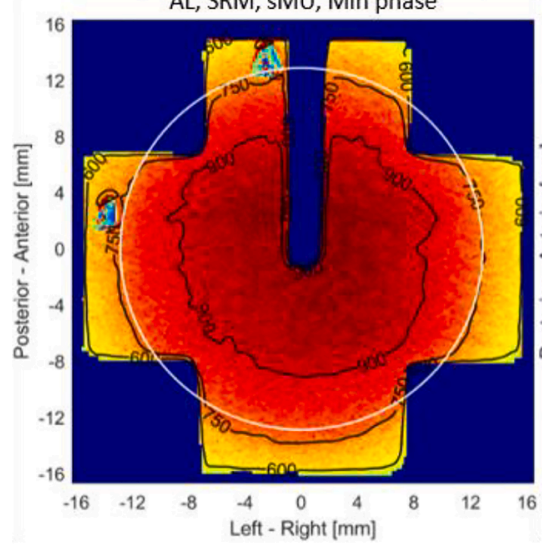

AL, SRM, mMU, Max phase

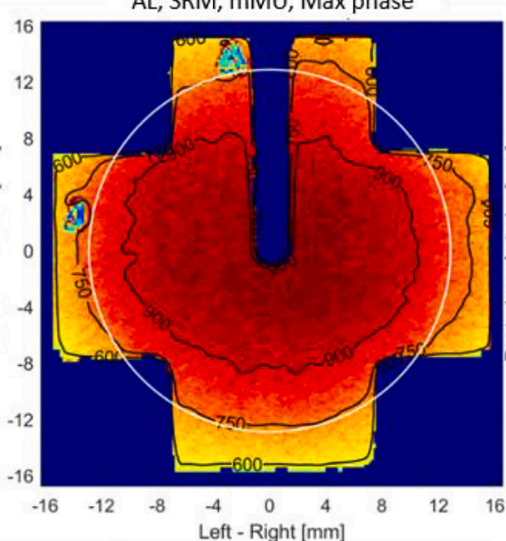

AL, SRM, mMU, Mid phase

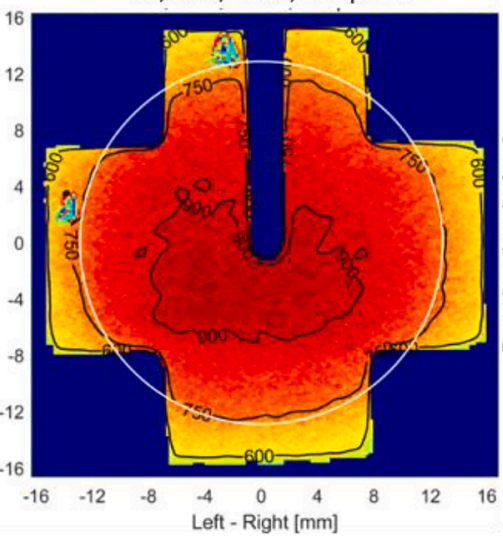

AL, SRM, mMU, Min phase

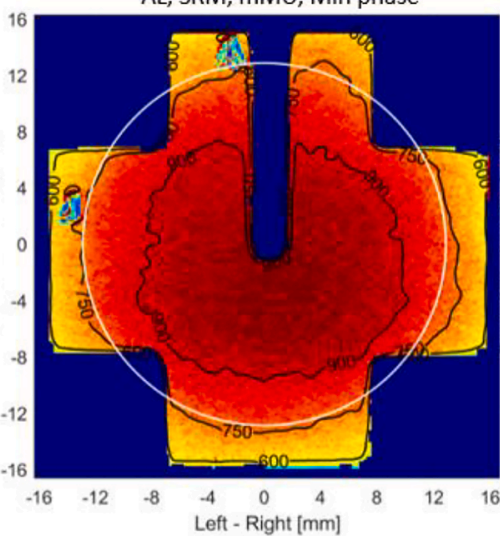

AL, SRM, IMU, Max phase

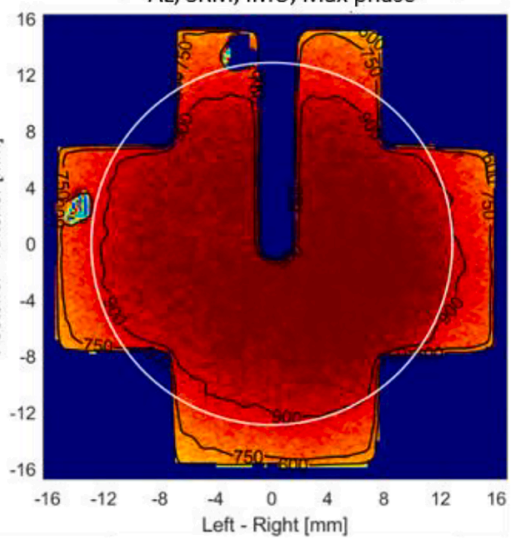

AL, SRM, IMU, Mid phase

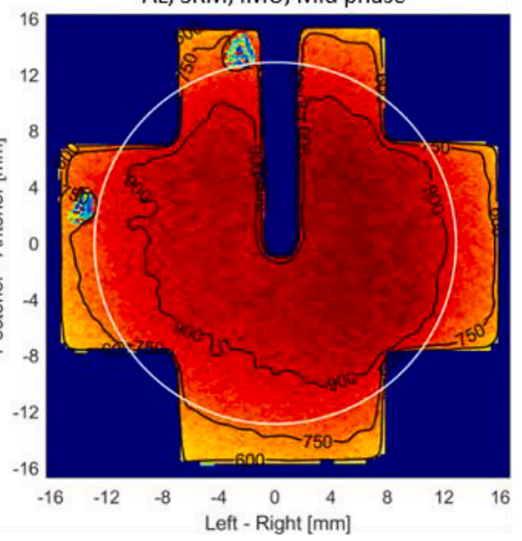

AL, SRM, IMU, Min phase

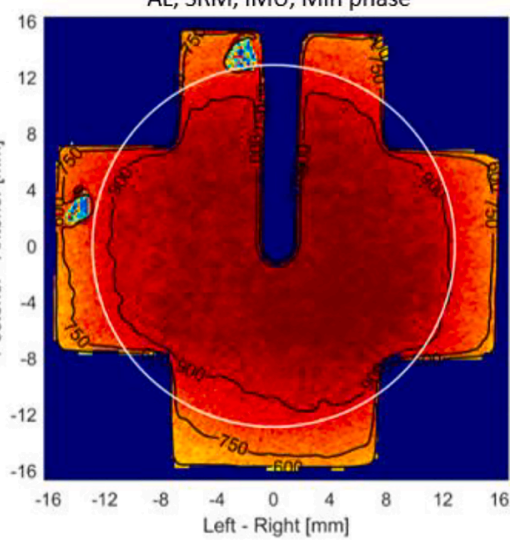

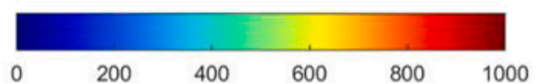

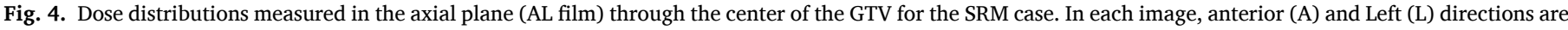

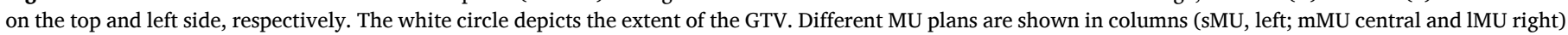

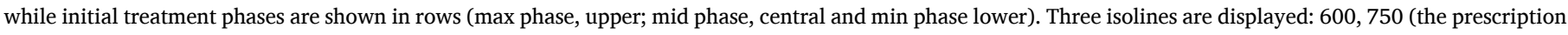
dose), and 900 cGy. Color table is in cGy.

Table 3, with the average value and standard deviation of three initial delivery phases. Generally, the SRM cases tended to have higher GTV coverage and less dependency on beam-on phases than the LRM cases. For SRM, the best GTV coverage was found with the IMU plan. For SRM, all different MU plans tested provided that the prescribed dose was at least delivered to $95 \%$ of the GTV. For LRM, the mMU plan presented the lowest GTV coverage and largest discrepancy between beam-on phases. sMU and IMU plans had similar GTV coverage for both AL and AS films, while the coverage was relatively independent from beam-on phase for the IMU plan only. For LRM, all different MU plans tested were not able to provide an average delivery of the prescribed dose to more than $95 \%$ of the GTV over the three fractions tested.

The conformity index for SRM and LRM cases for various MU plans and the initial delivery phase are shown in Table 4. For SRM, nCI increased along with the increased number of MUpb and showed little inter-fraction variation. For LRM, the averaged nCI varied slightly among different MU plans. Interestingly, the lMU plan provided a lower value of $\mathrm{nCI}$ as well as inter-fraction variation for LRM with respect to SRM. As observed for the target coverage for LRM, the mMU plan provided the worst results with the highest nCI. The nCI for AS films was 


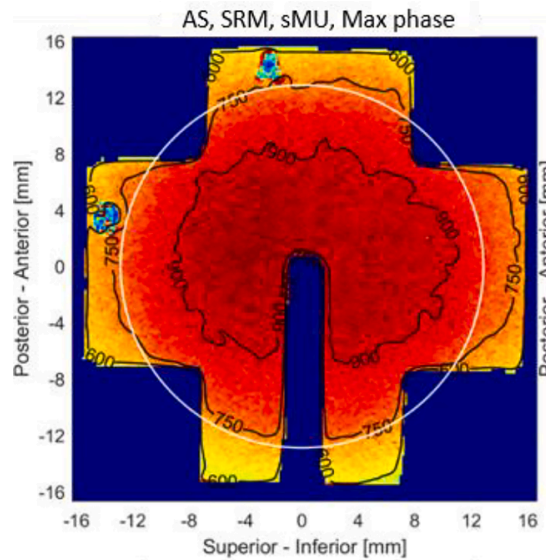

AS, SRM, sMU, Mid phase
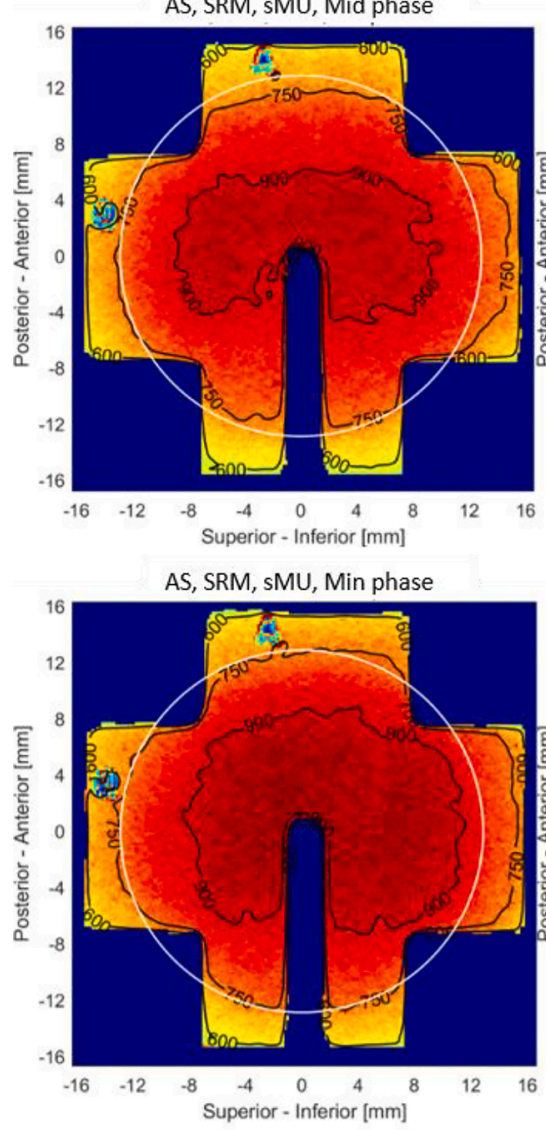

AS, SRM, mMU, Max phase

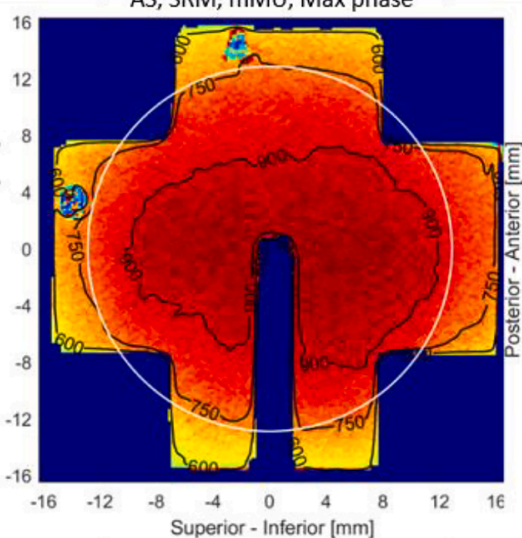

AS, SRM, mMU, Mid phase

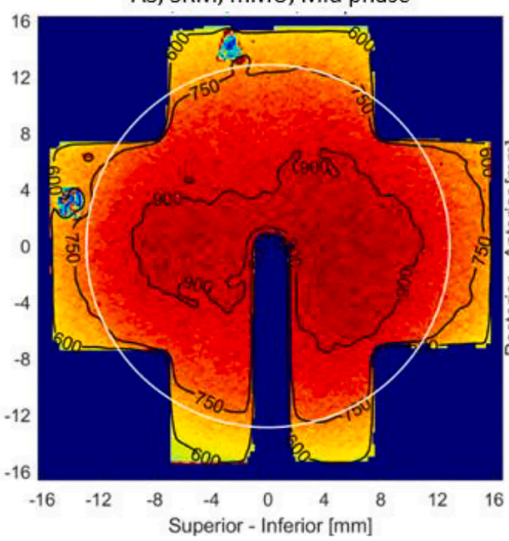

AS, SRM, mMU, Min phase

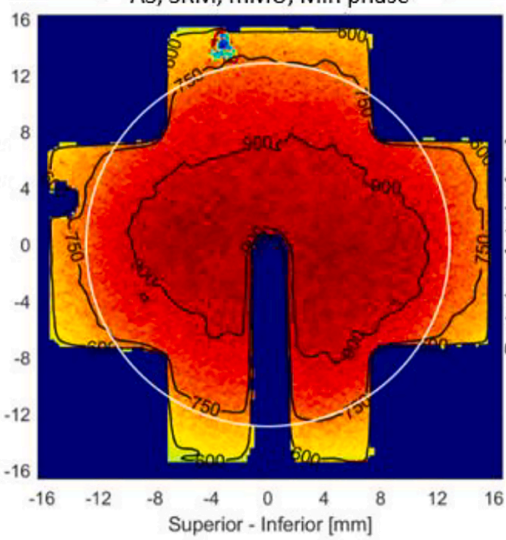

AS, SRM, IMU, Max phase

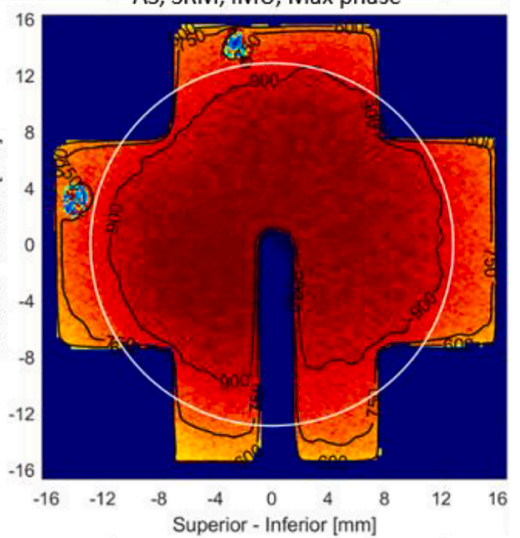

AS, SRM, IMU, Mid phase

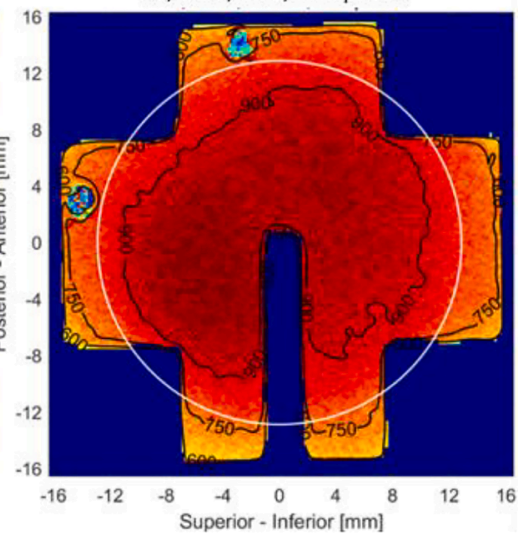

AS, SRM, IMU, Min phase

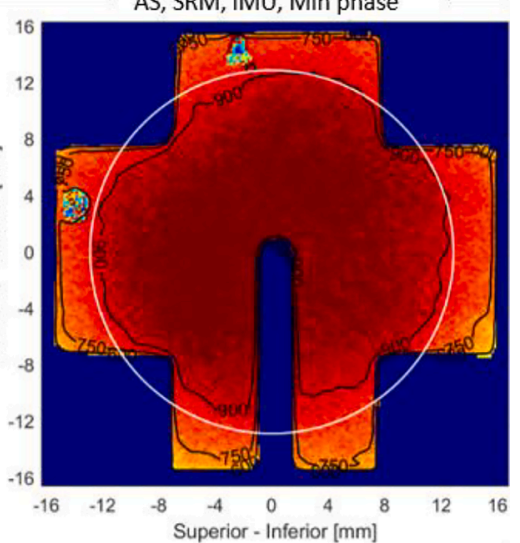

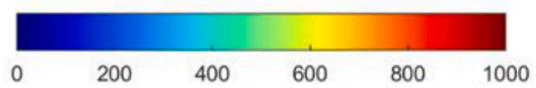

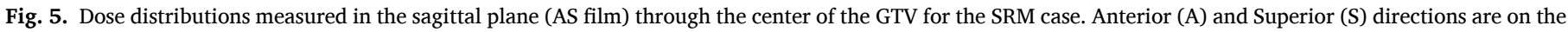

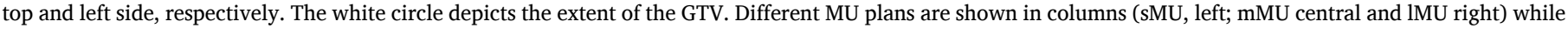

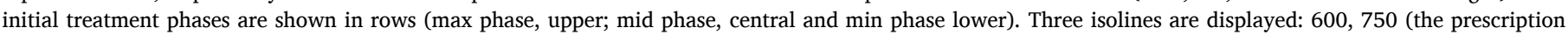
dose), and 900 cGy. Color table is in cGy.

found to be larger than for AL films for both respiratory motions due to the asymmetry of dose distribution in the SI direction.

\section{Discussion}

In this study, we investigated the magnitude of the interplay effect existing between the planned MU distribution of the CyberKnife system and a moving tumor for the clinical scenarios that may benefit from an indirect tracking focused on a static bone structure as an alternative to the real-time motion tracking.
We intentionally varied the MU distribution range in the planning to assess its impact on the interplay effect with two different respiratory motions programmed in a dynamic thoracic phantom.

Our results show that this specific interplay effect also occurred for small respiratory motion and its magnitude increased with the amplitude of the respiratory motion.

Evaluating the planned MU dose distributions provided us with a first indication as to how the VOLO ${ }^{\mathrm{TM}}$ optimization algorithm works in beam segmentation. As mentioned in the previous section, the number of maximum nodes (and thus beams) was kept fixed to its highest 

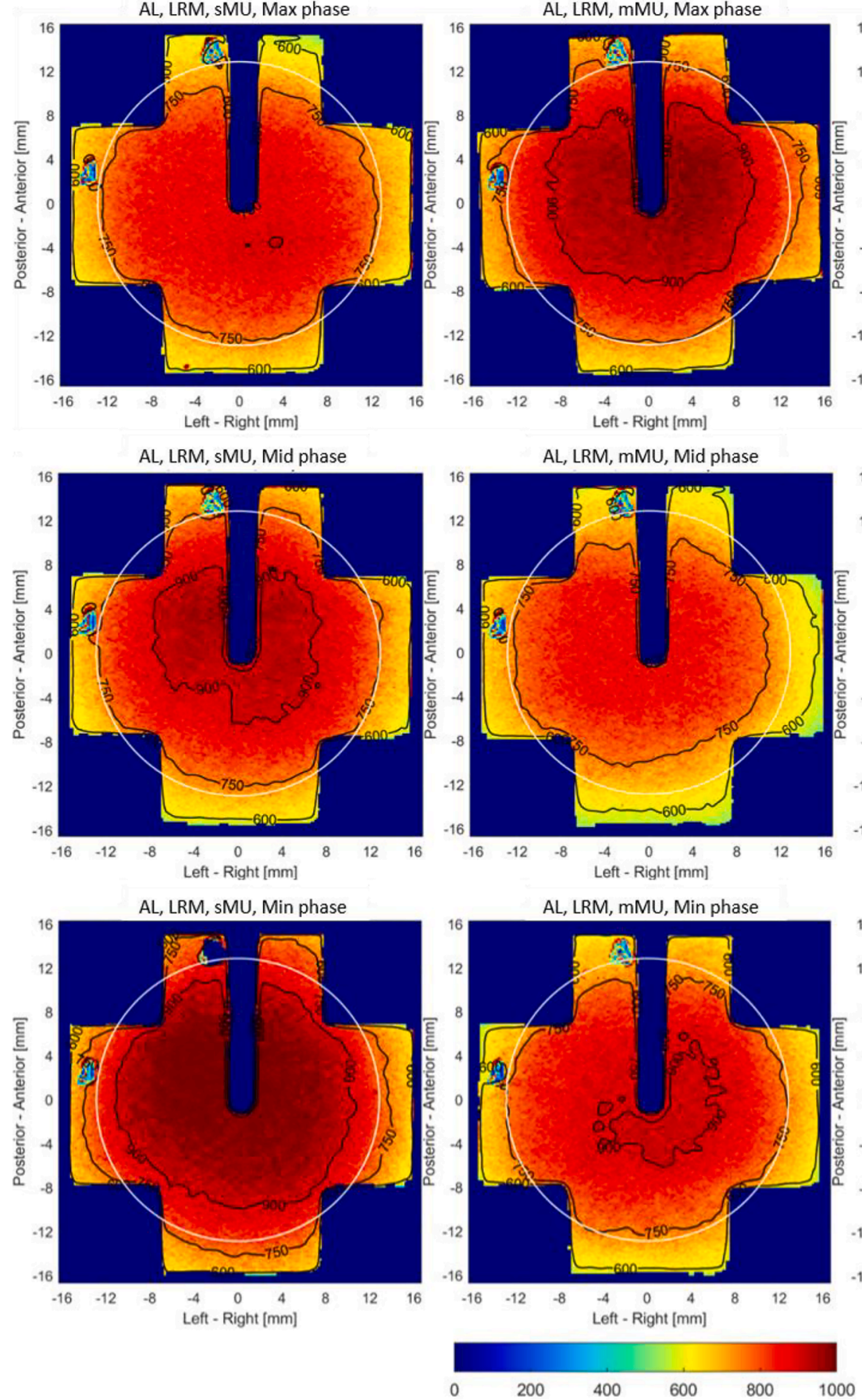

AL, LRM, mMU, Max phase

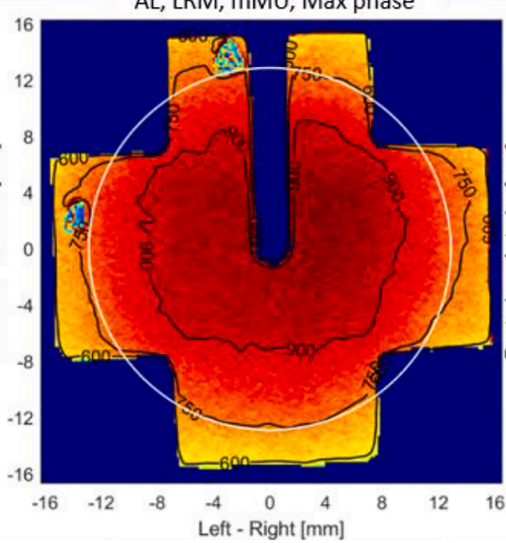

AL, LRM, mMU, Mid phase

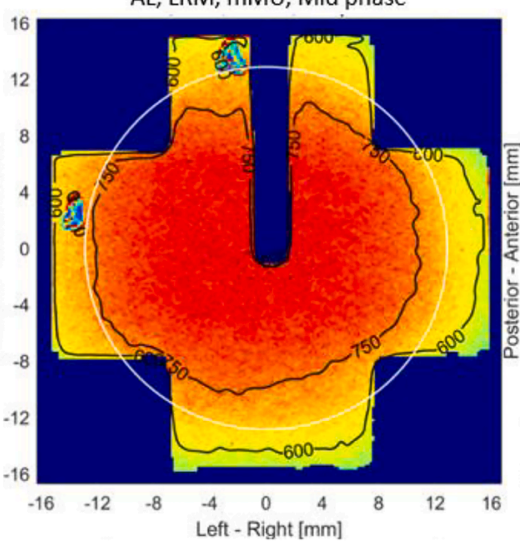

$\mathrm{AL}, \mathrm{LRM}, \mathrm{mMU}$, Min phase

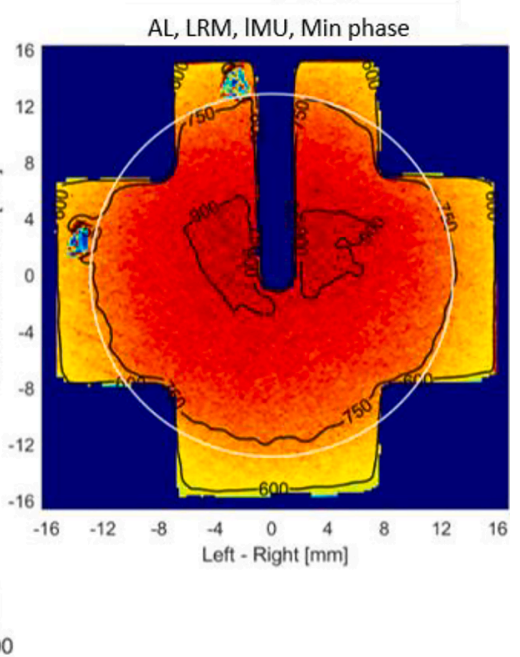

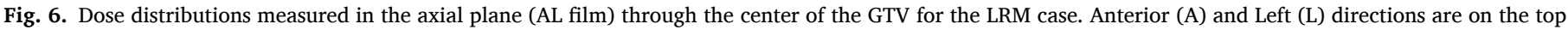

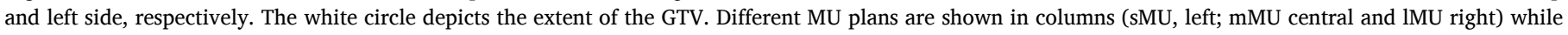

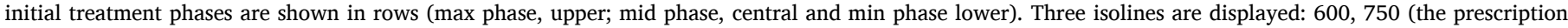
dose), and $900 \mathrm{cGy}$. Color table is in cGy.

available value among different MU plans. Nevertheless, for all plans this value was never reached, and for mMU and IMU plans in particular the number of beams was found to be very small. This could suggest a segmentation process in which the $\mathrm{MU}_{\mathrm{pb}}$ are maximized within the available range set by the user in order to deliver the treatment in the shortest time. When both minimum and maximum MU thresholds are increased, as was done in this study, the process led to a dramatic reduction of beams, in particular for SRM where the target dimensions were smaller. When a small number of beams is involved, this may lead to the reduction of dose conformity and the increase of hot spots around the tumor and at the beam entrances [25].

Despite the ITV approach used, that consisted also of 3 additional mm margin for PTV generation, the objective to cover $100 \%$ of the GTV with the prescription dose was never met. In fact, although the average GTV coverage was found to be better for SRM, it reached an acceptable value for the $1 \mathrm{MU}$ plan only at the cost of dose conformity. For lung tumors, our current approach for clinical cases involves the use of 6 minimum and 80-100 maximum $\mathrm{MU}_{\mathrm{pb}}$ per fraction as typical values. Although, on the one hand, this allows for a high number of beams involved and hence an optimal dose conformity, it would not always be, 

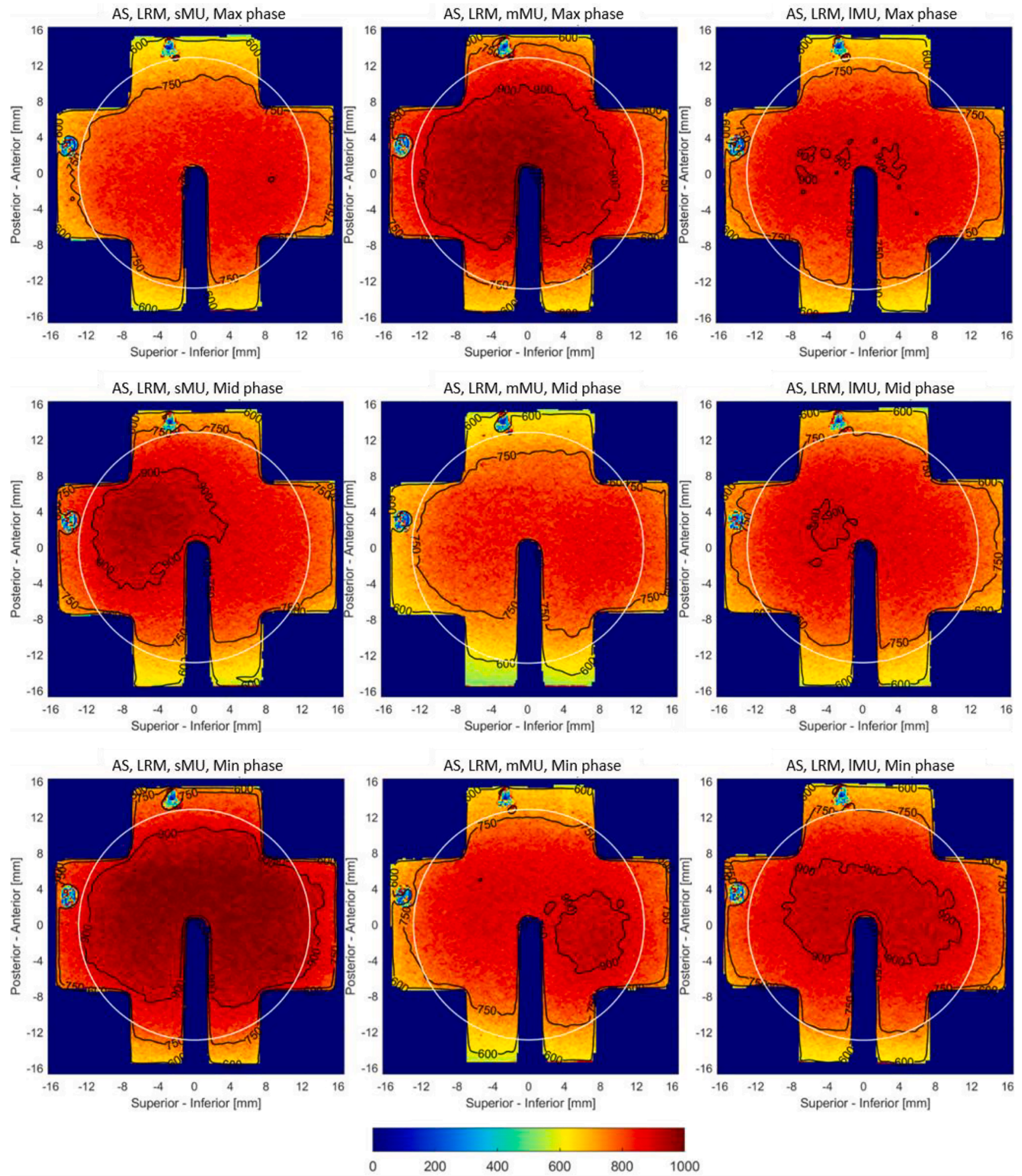

Fig. 7. Dose distributions measured in the axial plane (AS film) through the center of the GTV for the LRM case. Anterior (A) and Superior (S) directions are on the top and left side, respectively. The white circle depicts the extent of the GTV. Different MU plans are shown in columns (sMU, left; mMU central and lMU right) while initial treatment phases are shown in rows (max phase, upper; mid phase, central and min phase lower). Three isolines are displayed: 600,750 (the prescription dose), and 900 cGy. Color table is in cGy.

on the other hand, the best approach in terms of dose delivery robustness. In fact, the lMU plan was found to be the most robust in terms of intra- and inter-fraction delivery for SRM, suggesting that increasing the average of the MU plan distribution may overcome the geographical miss for small respiratory motions. Indeed, while for LRM a large intraand inter-fraction variation was expected, it was surprising to observe how the variation of the $\mathrm{MU}_{\mathrm{pb}}$ range had an impact on the dose delivery for SRM too. The same approach of increasing the $\mathrm{MU}_{\mathrm{pb}}$ range can also be used when large respiratory motion occurs. For LRM, target coverage was found to be similar for sMU and lMU plans. However, this latter plan provided less inter-fraction variability.

Although increasing the mean value of the MU distribution might enable a better GTV coverage and a reduced inter-fraction variation, the dose conformity unfortunately decreased due to the limited number of beams, in particular for SRM. The nCI of 1.33 measured for the IMU plan was close to the limit regarding acceptance criteria. This approach should be avoided for small lesions positioned near the chest wall to avoid any extra irradiation of the ribs and a consequent radio-induced 
Table 3

The GTV coverage (expressed in \% of volume receiving $7.5 \mathrm{~Gy}$ ) and standard deviation is listed according to the different breathing pattern and different MU plans.

\begin{tabular}{|c|c|c|c|c|c|c|c|}
\hline \multirow{2}{*}{\multicolumn{2}{|c|}{$\begin{array}{l}\text { Breathing } \\
\text { pattern } \\
\text { MUpb plan }\end{array}$}} & \multicolumn{3}{|l|}{ SRM } & \multicolumn{3}{|l|}{ LRM } \\
\hline & & \multirow{2}{*}{$\frac{\mathrm{sMU}}{97.8}$} & \multirow{2}{*}{$\frac{\mathrm{mMU}}{\mathrm{97.9}}$} & \multirow{2}{*}{$\frac{1 \mathrm{MU}}{99.9}$} & \multirow{2}{*}{$\frac{\text { sMU }}{85.9}$} & \multirow{2}{*}{$\frac{\mathrm{mMU}}{98.9}$} & \multirow{2}{*}{$\frac{\mathrm{IMU}}{96.2}$} \\
\hline $\mathrm{AL}$ & $\max$ & & & & & & \\
\hline & mid & 95.2 & 95.2 & 99.2 & 94.2 & 75.7 & 88.8 \\
\hline & $\min$ & 98 & 97.1 & 99.9 & 99.7 & 91.9 & 90.9 \\
\hline- & average & $\begin{array}{l}97 \pm \\
1.6\end{array}$ & $\begin{array}{l}96.7 \pm \\
1.4\end{array}$ & $\begin{array}{l}99.7 \pm \\
0.4\end{array}$ & $\begin{array}{l}93.3 \pm \\
6.9\end{array}$ & $\begin{array}{l}88.8 \pm \\
11.9\end{array}$ & $\begin{array}{l}92 \pm \\
3.8\end{array}$ \\
\hline \multirow[t]{4}{*}{ AS } & $\max$ & 96.5 & 97.6 & 99.9 & 89.1 & 98.5 & 93.7 \\
\hline & mid & 95.8 & 95 & 99.9 & 95.3 & 80.1 & 94 \\
\hline & $\min$ & 97.1 & 97.6 & 100 & 99.8 & 92.2 & 97.6 \\
\hline & average & $\begin{array}{l}96.5 \pm \\
0.7\end{array}$ & $\begin{array}{l}96.7 \pm \\
1.5\end{array}$ & $\begin{array}{l}99.93 \pm \\
0.06\end{array}$ & $\begin{array}{l}94.7 \pm \\
5.4\end{array}$ & $\begin{array}{l}90.3 \pm \\
9.4\end{array}$ & $\begin{array}{l}95.1 \pm \\
2.2\end{array}$ \\
\hline \multicolumn{2}{|c|}{$\begin{array}{l}\text { Overall } \\
\text { average }\end{array}$} & $\begin{array}{l}96.8 \pm \\
1.7\end{array}$ & $\begin{array}{l}96.7 \pm \\
1.5\end{array}$ & $\begin{array}{l}99.8 \pm \\
0.4\end{array}$ & $\begin{array}{l}94.0 \pm \\
8.8\end{array}$ & $\begin{array}{l}89.6 \pm \\
15.2\end{array}$ & $\begin{array}{l}93.6 \pm \\
4.4\end{array}$ \\
\hline
\end{tabular}

Table 4

Conformity number and standard deviation is listed according to the different breathing pattern and different MU plans.

\begin{tabular}{|c|c|c|c|c|c|c|c|}
\hline \multicolumn{2}{|c|}{$\begin{array}{l}\text { Breathing } \\
\text { pattern } \\
\text { MUpb plan }\end{array}$} & $\begin{array}{l}\text { SRM } \\
\text { sMU }\end{array}$ & \multirow{2}{*}{$\frac{\mathrm{mMU}}{1.12}$} & \multirow{2}{*}{$\frac{1 \mathrm{MU}}{1.30}$} & \multirow{2}{*}{$\begin{array}{l}\text { LRM } \\
\text { sMU } \\
1.18\end{array}$} & \multirow{2}{*}{$\frac{\mathrm{mMU}}{1.15}$} & \multirow{2}{*}{$\frac{1 \mathrm{MU}}{1.10}$} \\
\hline $\mathrm{AL}$ & $\max$ & 1.07 & & & & & \\
\hline & mid & 1.08 & 1.19 & 1.22 & 1.13 & 1.51 & 1.14 \\
\hline & $\min$ & 1.07 & 1.08 & 1.31 & 1.16 & 1.11 & 1.13 \\
\hline - & average & $\begin{array}{l}1.07 \pm \\
0.01\end{array}$ & $\begin{array}{l}1.13 \pm \\
0.05\end{array}$ & $\begin{array}{l}1.28 \pm \\
0.05\end{array}$ & $\begin{array}{l}1.15 \pm \\
0.03\end{array}$ & $\begin{array}{l}1.26 \pm \\
0.22\end{array}$ & $\begin{array}{l}1.13 \pm \\
0.02\end{array}$ \\
\hline \multirow[t]{4}{*}{ AS } & $\max$ & 1.13 & 1.19 & 1.30 & 1.25 & 1.23 & 1.20 \\
\hline & mid & 1.14 & 1.14 & 1.27 & 1.26 & 1.41 & 1.21 \\
\hline & $\min$ & 1.15 & 1.16 & 1.41 & 1.34 & 1.24 & 1.22 \\
\hline & average & $\begin{array}{l}1.14 \pm \\
0.01\end{array}$ & $\begin{array}{l}1.17 \pm \\
0.02\end{array}$ & $\begin{array}{l}1.33 \pm \\
0.07\end{array}$ & $\begin{array}{l}1.28 \pm \\
0.05\end{array}$ & $\begin{array}{l}1.29 \pm \\
0.10\end{array}$ & $\begin{array}{l}1.21 \pm \\
0.01\end{array}$ \\
\hline \multicolumn{2}{|c|}{$\begin{array}{l}\text { Overall } \\
\text { average }\end{array}$} & $\begin{array}{l}1.11 \pm \\
0.01\end{array}$ & $\begin{array}{l}1.15 \pm \\
0.06\end{array}$ & $\begin{array}{l}1.30 \pm \\
0.09\end{array}$ & $\begin{array}{l}1.22 \pm \\
0.06\end{array}$ & $\begin{array}{l}1.27 \pm \\
0.24\end{array}$ & $\begin{array}{l}1.17 \pm \\
0.02\end{array}$ \\
\hline
\end{tabular}

toxicity [26].

In particular for LRM, the mMU approach provided the worst results in terms of both target coverage and dose conformity. In order to provide a time-equivalent comparison with the respiratory cycle, given the CK dose rate of $900 \mathrm{MU} / \mathrm{min}$, the $\mathrm{mMU}$ plan had mean irradiation time per beam of approximately $4 \mathrm{~s}$ (see Table 2) while for the sMU and IMU plans it was 1.7 and 7.5 , respectively. Therefore, our results suggest that for large respiratory motion it may be preferable to use either an approach involving many beams with a short (less than the respiratory period) irradiation time or the opposite, meaning fewer beams with a long (more than the respiratory period) irradiation time. In fact, both sMU and IMU plans were found to be quite equivalent for LRM in terms of target coverage.

Overall, when static tracking is employed in treating moving tumors with the Cyberknife, we strongly recommend never reducing the PTV margins nor ignoring the ITV approach. Furthermore, the way of prescribing does should also be carefully considered. As previously mentioned, the dose was prescribed so that $98 \%$ of the GTV should have received the prescription dose calculated with a type $\mathrm{B}$ algorithm. As a consequence, the resulting median dose to the GTV was at least $1 \mathrm{~Gy}$ higher than the prescribed dose and, as demonstrated, this dose was not enough to guarantee the delivery of the prescribed dose to the whole GTV. For this specific clinical scenario, the prescription to the median of the GTV used for real-time tracking treatments [21,27] may end up being a sub-optimal choice that could introduce a systematic underdosage of the GTV. In fact, for gated treatments, deviations between planned and delivered dose only slightly affect the GTV coverage [28], while for non-gated delivery it may drop to $90 \%$ when pre-treatment CT verification are adopted [29]. Here, where the interplay effects are sensibly larger in magnitude and imaging verification does not take into account the tumor directly, the dose should be prescribed to the $100 \%$ of the GTV to ensure an appropriate dose delivery.

Obviously, this study has some limitations. Indeed, our results were affected by several other parameters (both planned and respiratorybased) apart from minimum $\mathrm{MU}_{\mathrm{pb}}$ such as collimator size and tumor motion range that could also affect the interplay effect. In this study, collimator sizes of $25 \mathrm{~mm}$ and $35 \mathrm{~mm}$ were adopted in the balance of conformity and efficiency. Our results confirmed a published study [15] that suggested the use of large collimators $(30 \mathrm{~mm})$ in treating moving lung tumors with XST, especially when small tumor motion range (less than $10 \mathrm{~mm}$ ) occurs.

Although our choice of collimator size seems reasonable considering the size of the GTV, different approaches may be used. In particular, smaller collimator sizes would have improved dose conformity at the cost of delivery time and potential increasing of intra-fraction motion. On the other hand, the use of larger collimator size would have produced moderate dose fall-off reducing the benefit of CK. In terms of tumor motion, in this study only two respiratory patterns were evaluated. An additional group of different respiratory patterns with different motion amplitudes and respiratory periods might benefit a more comprehensive discussion on the effect of respiratory motion. The inter-fraction dose variation observed over three fractions was significantly larger (up to ten times) for LRM than SRM. These results are in line with those published by Chan et al [15] who observed a linear increase of inter-fraction dose variation with respiratory motion in the SI direction.

As per the intrinsic nature of this in-phantom study, our results remain valid for regular motion patterns and for tumors that do not show any base-line shift. We do recommend to verify prior or ideally during every fraction motion amplitude by means of several available devices and the presence of any base-line tumor drifts through imaging. Our current practice is to check the consistency between respiratory signals carried out with the Anzai belt at the time of planning CT and of treatment. Although belts do not generally provide an absolute reference, their signal reproducibility may be improved by carefully positioning the sensor on the patient and optimized selection of the offset and gain parameters properly. Similarly, the base-line shift should be assessed prior every fraction to consider the planned ITV still adequate by means of 4DCT or fluoroscopy [29] It is recommended to treat moving lesions which are adjacent or in proximity to the spine where the surrogacy relationship is the best. In this way, both tumor and bone structure can be visualized in the orthogonal images providing information on their mutual position.

The results we have shown were extrapolated from two orthogonal films that may not represent the full picture of the GTV. It is possible that target coverage and dose conformity may also vary along other plans, providing better or worse results in terms of three-dimensional dosimetry. To overcome this limitation, the use of 3D polymer-based dosimetry could be implemented in commercially available dynamic phantoms. The limited size of the films also represented a limitation since the area outside of the GTV was too small to draw any conclusion in terms of dose delivered. Finally, the only moving part of the phantom was the tumor rod neglecting any possible spine motion that may introduce a further level of inaccuracy while treating real patient. The in-phantom accuracy of the XST of less than $1 \mathrm{~mm}$ has been shown to be similar in real patient [30]. However, when XST is employed for indirect lung tracking, on-line imaging should be performed at its maximum rate to minimize any further interplay effect due to systematic spine misalignement.

Despite these limitations, this study provides clear indications for a future planning strategy. The results have led us to reconsider our clinical practice; from now on we will privilege an increased mean of $\mathrm{MU}_{\mathrm{pb}}$ value for targets not placed close to the thoracic wall. 


\section{Conclusions}

This study confirmed the presence of the interplay effect between target motion and beam irradiation time when a static spine tracking technique was adopted in CyberKnife. The impact was non-negligible especially for large respiratory motion when the GTV coverage fell below $90 \%$, while it remained relatively independent of MU for small respiratory motion.

In general, large numbers of minimum $\mathrm{MU}_{\mathrm{pb}}$ should be used. This enables a maximization of the GTV coverage for small motion amplitude and, in turn, a minimization of the inter-fraction dose variation, for large motion amplitude.

\section{Declaration of Competing Interest}

The authors declare that they have no known competing financial interests or personal relationships that could have appeared to influence the work reported in this paper.

\section{Appendix A. Supplementary data}

Supplementary data to this article can be found online at https://doi. org/10.1016/j.ejmp.2021.08.016.

\section{References}

[1] Kilby W, Dooley JR, Kuduvalli G, Sayeh S, Maurer CR. The CyberKnife ${ }^{\circledR}$ robotic radiosurgery system in 2010. Technol Cancer Res Treat. 2010;9(5):433-52. https://doi.org/10.1177/153303461000900502.

[2] Murphy MJ. Tracking moving organs in real time. Semin Radiat Oncol. 2004;14(1): 91-100. https://doi.org/10.1053/j.semradonc.2003.10.005.

[3] Schweikard A, Glosser G, Bodduluri M, Murphy MJ, Adler JR. Robotic motion compensation for respiratory movement during radiosurgery. Comput Aided Surg. 2000;5(4):263-77. https://doi.org/10.3109/10929080009148894.

[4] Schweikard A, Shiomi H, Adler J. Respiration tracking in radiosurgery. Med Phys. 2004;31(10):2738-41. https://doi.org/10.1118/1.1774132.

[5] Yang Z-Y, Chang Yu, Liu H-Y, Liu G, Li Q. Target margin design for real-time lung tumor tracking stereotactic body radiation therapy using CyberKnife Xsight Lung tracking system. Sci Rep. 2017;7(1). https://doi.org/10.1038/s41598-017-11128W.

[6] Fu D, Kahn R, Wang B, Wang H, Mu Z, Park J, et al. In: Treating Tumors that Move with Respiration. Berlin, Heidelberg: Springer Berlin Heidelberg; 2007. p. 265-82. https://doi.org/10.1007/978-3-540-69886-9_26.

[7] Baumann R, Chan MKH, Pyschny F, et al. Clinical Results of Mean GTV Dose Optimized Robotic-Guided Stereotactic Body Radiation Therapy for Lung Tumors. Front Oncol. 2018;17(8):171.

[8] Kothary N, Heit JJ, Louie JD, et al. Safety and efficacy of percutaneous fiducial marker implantation for image-guided radiation therapy. J Vasc Interv Radiol. 2009;20(2):235-239. 3https://doi.org/10.1016/j.jvir.2008.09.026.

[9] Loo Jr BW, Kavanagh BD, Meyer JL. Motion management and image guidance for thoracic tumor radiotherapy: Clinical treatment programs. Front Radiat Ther Oncol. 2011;43:271-91.

[10] CyberKnife ${ }^{\circledR}$ Treatment Delivery System. Treatment Delivery Manual. Date of Revision 2018-06. Version 11.1.

[11] Bahig H, Campeau M-P, Vu T, Doucet R, Béliveau Nadeau D, Fortin B, et al. Predictive parameters of cyberknife fiducial-less (XSight Lung) applicability for treatment of early non-small cell lung cancer: A single-center experience. Int $\mathrm{J}$ Radiat Oncol Biol Phys. 2013;87(3):583-9. https://doi.org/10.1016/j. ijrobp.2013.06.2048.
[12] Bibault JE, Prevost B, Dansin E, et al. Radiotherapie stereotaxique de carcinomes bronchiques primitifs : suivi non invasif de la cible en temps réel. Cancer Radiother. 2010;14(8):690-7. https://doi.org/10.1016/j.canrad.2010.03.010.

[13] Stroom JC, Heijmen BJ. Geometrical uncertainties, radiotherapy planning margins, and the ICRU-62 report. Radiother Oncol. 2002;64(1):75-83. https://doi.org/ 10.1016/S0167-8140(02)00140-8.

[14] Bortfeld T, Jiang SB, Rietzel E. Effects of motion on the total dose distribution. Semin Radiat Oncol. 2004;14(1):41-51. https://doi.org/10.1053/j. semradonc.2003.10.011.

[15] Chan M, Kwong D, Lee V, Leung R, Wong M, Blanck O. Feasibility study of robotic hypofractionated lung radiotherapy by individualized internal target volume and XSight Spine Tracking: A preliminary dosimetric evaluation. J Cancer Res Ther. 2015;11(1):150. https://doi.org/10.4103/0973-1482.138220.

[16] Chang Y, Liu HY, Liang ZW, et al. Dosimetric effect of intrafraction tumor motion in lung stereotactic body radiotherapy using CyberKnife Static Tracking System. Technol Cancer Res Treat. 2019;18:1-10. https://doi.org/10.1177/ 1533033819859448370.

[17] Chan MKH, Kwong DLW, Ng SCY, Tong ASM, Tam EKW. Experimental evaluations of the accuracy of 3D and 4D planning in robotic tracking stereotactic body radiotherapy for lung cancers. Med Phys. 2013;40(4):041712. https://doi.org/ 10.1118/1.4794505.

[18] Xsight ${ }^{\circledR}$ Lung Tracking Phantom Kit \& 4D Planning Phantom, Models 18023-A, 18043 \& 18043-A. Quality assurance and E2E testing on Cyberknife ${ }^{\circledR}$ systems. CIRS, Computed Imaging Reference Systems, Inc. Norfolk, USA.

[19] Lujan AE, Larsen EW, Balter JM, Ten Haken RK. A method for incorporating organ motion due to breathing into 3D dose calculations. Med Phys. 1999;26(5):715-20. https://doi.org/10.1118/1.598577.

[20] Zeverino M, Marguet M, Zulliger C, Durham A, Jumeau R, Herrera F, et al. Novel inverse planning optimization algorithm for robotic radiosurgery: First clinical implementation and dosimetric evaluation. Phys Med. 2019;64:230-7. https://doi. org/10.1016/j.ejmp.2019.07.020.

[21] Lacornerie T, Lisbona A, Mirabel X, Lartigau E, Reynaert N. GTV-based prescription in SBRT for lung lesions using advanced dose calculation algorithms. Radiat Oncol. 2014;9(1). https://doi.org/10.1186/s13014-014-0223-5.

[22] Lewis D, Micke A, Yu X, Chan MF. An efficient protocol for radiochromic film dosimetry combining calibration and measurement in a single scan. Med Phys. 2012;39(10):6339-50. https://doi.org/10.1118/1.4754797.

[23] Zeverino M, Jaccard M, Patin D, Ryckx N, Marguet M, Tuleasca C, et al. Commissioning of the Leksell Gamma Knife ${ }^{\circledR}$ Icon $^{\mathrm{TM}}$. Med Phys. 2017;44(2): 355-63. https://doi.org/10.1002/mp.12052.

[24] Schlüter M, Schmitt D, Fürweger C, Schweikard A, Schlaefer A. (2020) Treatment Planning. In: Conti A., Romanelli P., Pantelis E., Soltys S., Cho Y., Lim M. (eds) CyberKnife NeuroRadiosurgery. Springer, Cham. https://doi.org/10.1007/978-3030-50668-15

[25] Cao Y, Zhu X, Ju X, Liu Y, Yu C, Sun Y, et al. Optimization of dose distributions of target volumes and organs at risk during stereotactic body radiation therapy for pancreatic cancer with dose-limiting auto-shells. Radiat Oncol. 2018;13(1). https://doi.org/10.1186/s13014-018-0956-7.

[26] Jumeau R, Filion É, Bahig H, Vu T, Lambert L, Roberge D, et al. A dosimetric parameter to limit chest wall toxicity in SABR of NSCLC. Br J Radiol. 2017;90 (1075):20170196. https://doi.org/10.1259/bjr.20170196.

[27] Lebredonchel S, Lacornerie T, Rault E, Wagner A, Reynaert N, Crop F. About the non-consistency of PTV-based prescription in lung. Phys Med. 2017;44:177-87. https://doi.org/10.1016/j.ejmp.2017.03.009.

[28] Zhao Bo, Yang Y, Li T, Li X, Heron DE, Huq MS. Dosimetric effect of intrafraction tumor motion in phase gated lung stereotactic body radiotherapy. Med Phys. 2012; 39(11):6629-37. https://doi.org/10.1118/1.4757916.

[29] Karlsson K, Lax I, Lindbäck E, Grozman V, Lindberg K, Wersäll P, et al. Estimation of delivered dose to lung tumours considering setup uncertainties and breathing motion in a cohort of patients treated with stereotactic body radiation therapy. Phys Med. 2021;88:53-64. https://doi.org/10.1016/j.ejmp.2021.06.015.

[30] Anthony KH, Dongshan F, Cristian C, et al. A Study of the Accuracy of CyberKnife Spinal Radiosurgery Using Skeletal Structure Tracking. Oper Neurosurg. 2007;60 (2):147-56. https://doi.org/10.1227/01.NEU.0000249248.55923.EC. 